\title{
Chorismate-Mutase-Catalyzed Claisen Rearrangement
}

Hong Guo and Niny Rao

\section{1}

\section{Introduction}

Chorismic acid is the key branch point intermediate in the biosynthesis of aromatic amino acids in microorganisms and plants (Scheme 1.1a) [1]. In the branch that leads to the production of tyrosine and phenylalanine, chorismate mutase (CM, chorismate-pyruvate mutase, EC 5.4.99.5) is a key enzyme that catalyzes the isomerization of chorismate to prephenate (Scheme 1.1b) with a rate enhancement of about $10^{6}-10^{7}$-fold. This reaction is one of few pericyclic processes in biology and provides a rare opportunity for understanding how Nature promotes such unusual transformations. The biological importance of the conversion from chorismate to prephenate and the synthetic value of the Claisen rearrangement have led to extensive experimental investigations [2-43].

In addition, the reaction catalyzed by chorismate mutase is a paradigm for the study of enzyme mechanism and has been a subject of extensive computational investigations [44, 47-83]. One of the main reasons for the current focus on the mechanism of this enzyme is the fact that the reaction is a straightforward unimolecular rearrangement of the substrate with no chemical transformations in the enzyme or the solvent during the reaction. This eliminates many of the problems that arise for other cases and may help to settle some of the long-standing issues concerning the origin of the catalysis [84].

Experimental results for the CM-catalyzed and uncatalyzed reaction, as well as structural information for chorismate mutase, have been extensively discussed in two previous reviews [2, 3]. There has been a rapid growth of literature in computational studies of chorismate mutase in the last few years. In this chapter, we shall begin by summarizing some key experimental data related to the Claisen rearrangement along with existing structural information for chorismate mutase. We will then review the results of computational studies of chorismate mutase and discuss different proposals that have been suggested for the mechanism of the CM-catalyzed reaction. 
a)<smiles>C=C(OC1C=C(C(=O)[O-])C=C[C@H]1O)C(=O)[O-]</smiles><smiles>C1CCCC1</smiles><smiles>O=C(Cl)CC1(C(=O)[O-])C=CC(O)C=C1</smiles><smiles>C#CC</smiles><smiles>N[C@@H](Cc1ccc(O)cc1)C(=O)O</smiles><smiles>N[C](Cc1ccccc1)C(=O)O</smiles>

chorismate

prephenate

tyrosine

phenylalanine

anthranilate
synthase

anthranilate<smiles></smiles>

tryptophan

b)<smiles>O=Cc1ccc(O)c(OC(=O)O)c1</smiles>

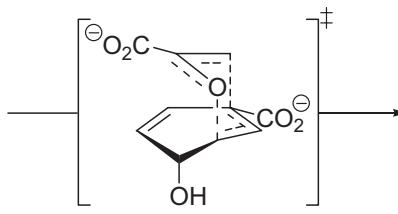<smiles>O=C([O-])CC1(C(=O)[O-])C=CC(O)C=C1</smiles>

Scheme 1.1

1.2

Experimental Studies

\subsection{1}

\section{Substrate Binding}

Knowles and coworkers $[13,14]$ demonstrated that the rearrangement of chorismate to prephenate proceeds through the same transition state (1, TS in Scheme 1.2) in solution and at the enzyme active site. The atoms of the [3,3]-pericyclic region in this TS are arranged in a "chair-like" configuration. The result of Knowles and coworkers has led to the suggestion that the bond breaking and making process starts from a chair-like pseudodiaxial conformer of chorismate (2, CHAIR in Scheme 1.2), where $C_{1}$ and $C_{9}$ are positioned to form the carbon-carbon bond, as required for the Claisen rearrangement. Thus, one straightforward way for chorismate mutase to catalyze the rearrangement is to bind the CHAIR conformer preferentially from solution and then catalyze its chemical transformation at the active site. A requirement for such a mechanism is a sufficiently large population of the CHAIR conformer in solution. To determine the population of CHAIR in solution, Copley and Knowles [15] measured the temperature variation of the ${ }^{1} \mathrm{H}$ coupling constants for the protons in the ring of chorismate. It was shown that al- 
though the dominant conformer(s) is a pseudodiequatorial conformation (see 3 of Scheme 1.2 for a schematic diagram), a pseudodiaxial conformer(s) exists at a reasonable level ( 12\%) in solution. Copley and Knowles [15] assumed that the pseudodiaxial conformer they observed in the NMR experiment was the CHAIR conformer and concluded that the enzyme could bind this reactive conformer directly from solution and catalyze its chemical transformation at the active site. But a later study of the transferred nuclear Overhauser effects for chorismate by Hilvert and his coworkers [17] failed to find evidence for the existence of CHAIR in solution. Recent molecular dynamics (MD) simulations [82, 83] suggested that the NMR data could correspond to other pseudodiaxial conformer(s) rather than CHAIR (see below).

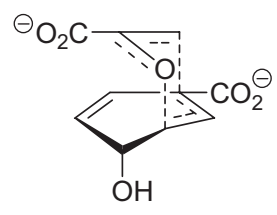

TS(1)

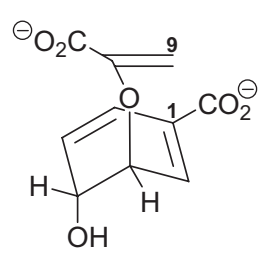

CHAIR(2)

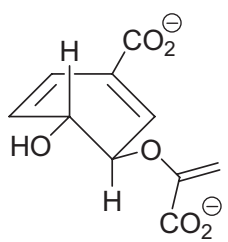

$\operatorname{DIEQ}(3)$<smiles>O=C([O-])[C@@H]1C=C[C@H]2C[C@H]1[C@@H](C(=O)[O-])C[C@H]2O</smiles>

TSA(4)

Scheme 1.2

\section{2 .2}

\section{Substrate Structural Requirements for Catalysis}

The structural features of the substrate required for binding and catalysis by Escherichia coli chorimate mutase (P-protein EcCM) and Bacillus subtillus chorismate mutase (BsCM) have been studied [22, 23]. Besides the allyl vinyl ether, the two carboxylic acid groups in chorismic acid were found to be very important for the catalysis. For instance, experimental studies [22] showed that ester 5 (see Scheme 1.3) was not a substrate or inhibitor for EcCM, suggesting that the presence of the sidechain carboxyl group is crucial for the binding and catalysis. EcCM and BsCM were also unable to catalyze the rearrangement of $\mathbf{6}$ (which lacks the ring carboxylic acid group) [22, 23], even though 6 proved to be a weak to modest competitive inhibitor ( $K_{\mathrm{i}}$ of 6 is $0.4 \mathrm{mM}$ and $0.5 \mathrm{mM}$ for EcCM and BsCM, repectively; for chorismate $K_{\mathrm{m}}$ is $0.32 \mathrm{mM}$ and $0.28 \mathrm{mM}$, respectively). Thus, the existence of the ring carboxyl group is also essential for the catalysis, but may not<smiles>[R]C(=C)OC1C=C([R])C=C[C@@H]1[R]</smiles>

$$
\begin{aligned}
& \text { 5: } \mathrm{R}_{1}=\mathrm{CO}_{2} \mathrm{H} ; \mathrm{R}_{2}=\mathrm{CO}_{2} \mathrm{Me} ; \mathrm{R}_{3}=\mathrm{OH} \\
& \text { 6: } \mathrm{R}_{1}=\mathrm{H} ; \mathrm{R}_{2}=\mathrm{CO}_{2} \mathrm{H} ; \mathrm{R}_{3}=\mathrm{OH} \\
& \text { 7: } \mathrm{R}_{1}=\mathrm{CO}_{2} \mathrm{H} ; \mathrm{R}_{2}=\mathrm{CO}_{2} \mathrm{H} ; \mathrm{R}_{3}=\mathrm{OMe}
\end{aligned}
$$

Scheme 1.3 
be required for the binding. Analog 7 was a reasonable substrate for EcCM $\left(K_{\mathrm{m}}=1.9 \mathrm{mM}\right.$ and $\left.k_{\text {cat }}=0.56 \mathrm{~s}^{-1}\right)$ with a rate acceleration $\left(k_{\text {cat }} / k_{\text {uncat }}\right)$ of $2 \times 10^{4}$ by the enzyme; for chorismic acid $k_{\text {cat }} / k_{\text {uncat }}=2 \times 10^{6}$. Thus, the free hydroxyl group at $\mathrm{C}_{4}$ may not be required for the catalysis by EcCM, but it is not clear whether this is also the case for BsCM (see below).

\section{2 .3}

\section{X-ray Structures of Chorismate Mutase}

A number of X-ray structures for chorismate mutase are available. The structures of BsCM and Saccharomyces cerevisiae (yeast) CM complexed with an endo-oxabicyclic transition state analog inhibitor (4, TSA in Scheme 1.2) [19] have been determined by Lipscomb and coworkers [6, 7, 12, 34]; the structures without TSA bound were also obtained for BsCM and yeast $\mathrm{CM}$ as well as for some of their mutants $[8,10,12,31]$. The X-ray structures for the monofunctional amino-terminal chorismate mutase domain engineered from the P-protein (EcCM) and a less active catalytic antibody 1 F7 complexed with TSA have been determined by Lee et al. [4] and Haynes et al. [33], respectively. Both EcCM and yeast CM are homodimers, whereas BsCM is a homotrimer. It has been demonstrated that the dimer of EcCM can be superimposed onto a monomer of yeast CM [4, 11, 34], indicating a common evolutionary origin of the two CMs with an ancestral protein that was structurally closer to EcCM than to yeast CM [34]. Moreover, there was a possible gene duplication event in the evolution of yeast CM [34], allowing the formation of the regulatory domain for this enzyme. The structure of $\mathrm{BsCM}$, which consists mainly of $\beta$-sheets, is different from the almost all-helical structures of EcCM and yeast CM.

Scheme 1.4 shows the schematic diagrams for the active site structures of EcCM [4], yeast CM [12, 34], BsCM [6, 7] and catalytic antibody 1F7 [33]. The active site of BsCM is somewhat open and more solvent accessible than the more buried catalytic packets in EcCM and yeast CM. As is evident from Scheme 1.4a and b, most of the active site residues in EcCM and yeast CM are conserved. For instance, in the both cases the guanidinium groups of two Arg residues (Arg28 and Arg11' in EcCM and Arg16 and Arg157 in yeast CM, respectively) form salt bridges with the carboxylate groups of the inhibitor. Lys39(Lys168) in EcCM (yeast CM) is in hydrogen bond distances to the sidechain carboxylate group and the ether oxygen of TSA. A major difference between the two active sites is that the other residue interacting with the ether oxygen is Gln in $\mathrm{EcCM}$ (Gln88), but is Glu in yeast CM (Glu246). It has been shown that Glu246 has to be protonated for functionality of yeast CM. The replacement of Glu246 by Gln changes the $\mathrm{pH}$ optimum for the activity from a narrow to a broad $\mathrm{pH}$ range, even though the kinetic parameters are not significantly affected by the mutation (e.g., the effect on $k_{\text {cat }} / K_{\mathrm{m}}$ is less than 10-fold) [28]. Consistent with these observations on yeast CM, the replacement of Gln88 in EcCM by Glu leads to loss of activity of 700-fold at pH 7.5, but the activity of the Gln88Glu mutant can be reduced almost $10^{3}$-fold by simply lowering the $\mathrm{pH}$ to 4.9 [27] (see Table 1.1 and the next section for more details on the effects of mutations). 
a)

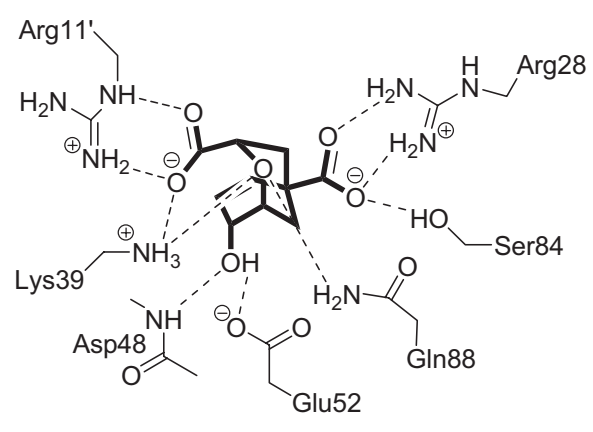

c)

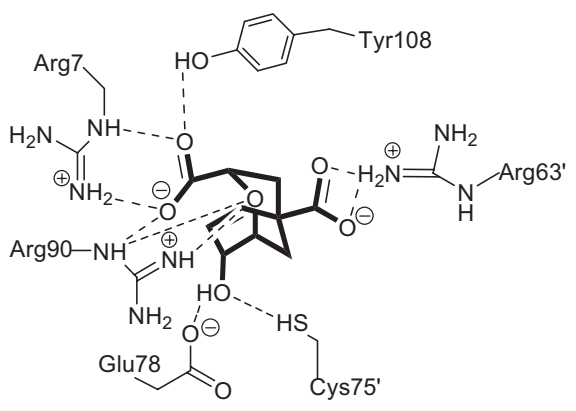

b)

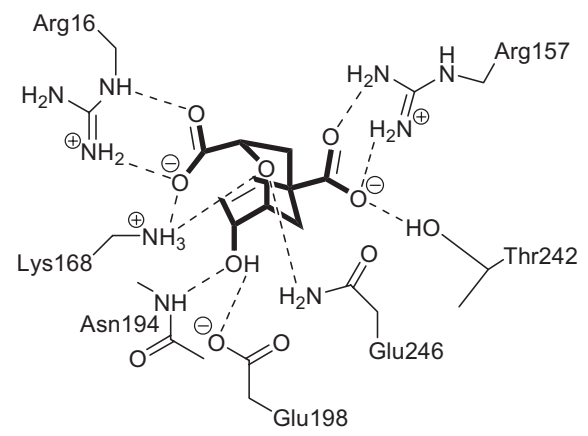

d)

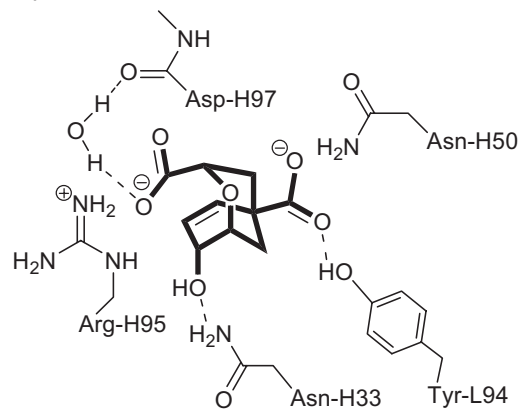

Scheme 1.4 The active sites of the CM complexes, (a) EcCM;

(b) yeast $\mathrm{CM}$; (c) BsCM; (d) catalytic antibody $1 \mathrm{~F} 7$.

Scheme $1.4 \mathrm{c}$ shows that the active site of $\mathrm{BsCM}$ also consists of highly charged residues. Arg7 forms a similar salt bridge with the sidechain carboxylate group of TSA as Arg11' in the EcCM complex. Arg90 interacts with the both ether oxygen and sidechain carboxylate group. Arg63 was not visible in the electron-density map in an earlier X-ray structure determination [7]. But a more recent X-ray structure [8] of higher resolution (1.3 $\AA$ ) without TSA bound showed that Arg63 is turned inward toward the active site and may therefore interact with the ring carboxylate group of TSA. Another interaction that exists in all the three CMs is the hydrogen bond between the $\mathrm{C}_{4}$-hydroxyl group of TSA and a Glu residue (Glu52 in EcCM, Glu198 in yeast CM and Glu78 in BsCM). This Glu residue appears to play a more important role for the reaction catalyzed by BsCM than by EcCM (see below). Comparison of the active site structures of EcCM, BsCM and yeast CM with that of the catalytic antibody (1F7) (Scheme 1.4d) shows that the enzymes provide many more hydrogen bonding and electrostatic interactions to the functional groups of TSA than does the antibody. The lack of the multiple interactions is believed to be responsible for the observed $10^{4}$-times lower activity of the antibody relative to that of the natural chorismate mutase [33]. 
1.2 .4

Effects of Mutations

For the uncatalyzed Claisen rearrangement $k_{\text {uncat }}$ is about $10^{-5} \mathrm{~s}^{-1}[20,31]$, and the $k_{\text {cat }}$ value for the CM-catalyzed reaction is approximately $46-72 \mathrm{~s}^{-1}$ (Table 1.1). Thus, the enzyme is able to accelerate the rate of the reaction by $10^{6}$ to $10^{7}$-fold. To identify the key residues that play an important role in the catalysis, a number of active site mutants were generated and characterized for EcCM [27, 35], yeast CM [28] and BsCM $[25,29,31,36]$ and the effects of mutations on the activity have been determined.

For EcCM, Arg28, Arg11' and Lys39 are involved in the direct interactions with the two carboxylate groups as well as the ether oxygen of TSA in the X-ray structure (Scheme 1.4a). Table 1.1 shows that these positively charged residues play a very important role in the catalysis [27]. For instance, the $k_{\text {cat }} / K_{\mathrm{m}}$ values for the Arg28Lys and Arg11Lys are approximately $10^{3}$ lower than wild-type, whereas the values for Lys39Ala and Lys39Arg are about $10^{4}$ lower. Similar observations were made for the related yeast CM, where the Arg157Ala, Arg16Ala and Lys168Ala mutants showed no detectable chorismate mutase activity [28]. The hydrogen bond between Gln88 (Glu246 in yeast CM) and the ether oxygen was also found to be very important. For instance, the replacement of Gln 88 by Ala leads to a reduction of the activity by $10^{4}$-fold. For the Glu52 mutants, the order of activity is Glu52 > Gln52 > Asp52 > Ala52. Glu52 interacts with the $\mathrm{C}_{4}$-hydroxyl group in the $\mathrm{X}$-ray structure. The higher activity of Glu52Gln than Glu52Asp seems to indicate that the existence of a carboxylate group in the vicinity of the $\mathrm{C}_{4}$-hydroxyl may not be necessary. This seems to be consistent with the earlier discussions of substrate structural requirements for the catalysis where it was shown that the free hydroxyl group at $\mathrm{C}_{4}$ may not be required in the case of the EcCM-catalyzed reaction (see above).

The kinetic parameters for BsCM mutants are also available [25, 29, 31, 36] and listed in Table 1.1. Arg7, which forms a similar interaction with TSA as Arg11' in EcCM, was found to be very important. For instance, the replacement of Arg7 by Ala leads to an approximately $5 \times 10^{5}$-fold reduction in $k_{\text {cat }} / K_{\mathrm{m}}$. Arg90, which interacts with the both ether oxygen and sidechain carboxylate group (Scheme 1.4c), is also crucial for the catalysis. For instance, the $k_{\text {cat }}$ and $k_{\text {cat }} / K_{\mathrm{m}}$ values for Arg90Gly are more than five orders of magnitude lower than those of the wild-type enzyme. Moreover, the importance of the positive charge on Arg90 was demonstrated by Hilvert and coworkers [36] who showed that there is a significant reduction of the activity $\left(>10^{4}\right.$-fold in $\left.k_{\text {cat }}\right)$ when Arg90 was replaced by citrulline, an isosteric but neutral arginine analog. Interestingly, the double mutants Cys88Lys/Arg90Ser and Cys88Ser/Arg90Lys restore a factor of more than $10^{3}$ in $k_{\text {cat }}$ compared to Arg90Gly [31]. Another important residue for the catalysis is Glu78. Glu78 is in a similar location as Glu52 in EcCM. Table 1.1 shows that the $k_{\text {cat }} / K_{\mathrm{m}}$ values for Glu78Ala and Glu78Gln are about $10^{4}$ lower than wild-type. By contrast, the activity of Glu78Asp is only 30 -fold lower. This seems to suggest that the existence of a carboxylate group in the vicinity of the $\mathrm{C}_{4}$-hydroxyl is more important for the 
1.2 Experimental Studies $\mid 7$

Table 1.1 Kinetic constants for EcCM and BsCM mutants.

\begin{tabular}{|c|c|c|c|c|c|c|}
\hline Enzyme & Mutant & $\begin{array}{l}k_{\text {cat }} \\
\left(\mathrm{s}^{-1}\right)\end{array}$ & $\begin{array}{l}K_{\mathrm{m}} \\
(\mu \mathrm{M})\end{array}$ & $\begin{array}{l}k_{\mathrm{cat}} / K_{\mathrm{m}} \\
\left(\mathrm{M}^{-1} \mathrm{~s}^{-1}\right)\end{array}$ & $\begin{array}{l}K_{\mathrm{i}} \text { for } 4 \\
(\mu \mathrm{M})\end{array}$ & Reference \\
\hline \multirow[t]{13}{*}{$\mathrm{EcCM}$} & Wild type & 72 & 296 & $2.4 \times 10^{5}$ & 3.66 & [27] \\
\hline & $\mathrm{R} 11 \mathrm{~A}$ & & $>2000$ & 26 & & [27] \\
\hline & $\mathrm{R} 11 \mathrm{~K}$ & & $>2000$ & 230 & & {$[27]$} \\
\hline & $\mathrm{R} 28 \mathrm{~A}$ & & $>2000$ & 170 & & {$[27]$} \\
\hline & $\mathrm{R} 28 \mathrm{~K}$ & & $>2000$ & 230 & & {$[27]$} \\
\hline & K39A & & $>2000$ & 4.3 & & {$[27]$} \\
\hline & K39R & & $>2000$ & 1.9 & & {$[27]$} \\
\hline & E52A & 0.49 & 4580 & 110 & 218 & {$[27]$} \\
\hline & E52D & 3.1 & 1440 & $2.2 \times 10^{3}$ & 78.4 & {$[27]$} \\
\hline & E52Q & 24 & 1080 & $2.3 \times 10^{4}$ & 26.8 & {$[27]$} \\
\hline & Q88A & & $>2000$ & 12 & & [27] \\
\hline & Q88E & & $>2000$ & 361 & & {$[27]$} \\
\hline & $\mathrm{Q} 88 \mathrm{E}(\mathrm{pH} 4.9)$ & 72 & 296 & $2.4 \times 10^{5}$ & 3.66 & [27] \\
\hline \multirow[t]{13}{*}{ BsCM } & Wild type & 46 & 67 & $6.9 \times 10^{5}$ & 3 & {$[36]$} \\
\hline & R90G & $2.7 \times 10^{-4}$ & 150 & 31 & & {$[31]$} \\
\hline & R90K & & & 31 & & {$[25]$} \\
\hline & R90A & & & $<1$ & & {$[25]$} \\
\hline & R7K & & & 717 & & {$[25]$} \\
\hline & R7A & & & 1 & & {$[25]$} \\
\hline & C75D/E78A & & & $1.66 \times 10^{3}$ & & {$[25]$} \\
\hline & E78D & 35.7 & 1297 & $2.75 \times 10^{4}$ & 43.6 & {$[25]$} \\
\hline & E78Q & & & 75 & & {$[25]$} \\
\hline & E78A & & & 33 & & {$[25]$} \\
\hline & C88K/R90K & 0.29 & 4300 & 67 & $>>1000$ & {$[31]$} \\
\hline & C88K/R90S & 0.32 & 1900 & 170 & 1100 & [31] \\
\hline & R90Cit & 0.0026 & 270 & 230 & 6.8 & {$[36]$} \\
\hline
\end{tabular}


BsCM-catalyzed reaction than for the EcCM-catalyzed reaction. Consistent with this suggestion, the activity of the Glu78Ala mutant is rescued 50 -fold by replacing $\mathrm{C} 75$ (which is also near the $\mathrm{C}_{4}$-hydroxyl group) with Asp in double mutant Glu78Ala/C75Asp [25]. The studies of substrate structural requirements for the catalysis (see above) showed that $\mathbf{6}$, which lacks the ring carboxylate group, is not a substrate for EcCM [22] and BsCM [23]. For EcCM, the residue that interacts with the ring carboxylate group is Arg 28, and the replacement of Arg28 by another residue leads to a significant reduction of the activity. However, for BsCM the corresponding residue has not been clearly identified. A recent X-ray structure [8] for BsCM suggested that Arg63 may interact with the ring carboxylate group, but the mutagenesis study for the Arg63 mutants has not been available.

\section{2 .5}

\section{Activation Parameters}

The activation parameters for the CM-catalyzed and uncatalyzed Claisen rearrangement are listed in Table $1.2[20,21,26,42]$. For the uncatalyzed reaction, the activation barrier $\left(\Delta G^{+}\right)$is $24.5 \mathrm{kcal} / \mathrm{mol}$. Chorismate mutase is able to reduce the activation barrier by $7-10 \mathrm{kcal} / \mathrm{mol}$. Table 1.2 shows that the rate acceleration is due to a reduction in the entropy of activation to near zero and a decrease in the enthalpy of activation by about $5 \mathrm{kcal} / \mathrm{mol}$; the only exception is the BsCM-catalyzed reaction for which there is a significant unfavorable $\Delta S^{\dagger}$. However, the reliability of these data has been called into question [44], and it was suggested [44] that both the substrate binding and product leaving are expected to show large solvent compensation effects involving $\Delta H^{\dagger}$ and $\Delta S^{\dagger}[45,46]$.

Table 1.2 Activation parameter for the catalyzed and uncatalyzed reaction ${ }^{\text {a) }}$.

\begin{tabular}{lllll}
\hline Enzyme & $\begin{array}{l}\Delta \boldsymbol{H}^{*} \\
\left(\mathrm{kcal} \cdot \mathrm{mol}^{-1}\right)\end{array}$ & $\begin{array}{l}\Delta \boldsymbol{S}^{*} \\
(\mathbf{e . u .})\end{array}$ & $\begin{array}{l}\Delta \boldsymbol{G}^{*} \\
\left(\mathrm{kcal} \cdot \mathrm{mol}^{-1}\right)\end{array}$ & $\begin{array}{l}\Delta \boldsymbol{\Delta} \mathbf{G}^{*} \\
\left(\mathbf{k c a l} \cdot \mathrm{mol}^{-1}\right)\end{array}$ \\
\hline BsCM & 12.7 & -9.1 & 15.4 & -8.9 \\
EcCM & 16.3 & -3.0 & 17.2 & -7.3 \\
K. pneumoniae & 15.9 & -1.1 & 16.2 & -8.3 \\
S. aureofaciens & 14.5 & -1.6 & 15.0 & -9.5 \\
Uncatalyzed & 20.5 & -12.9 & 24.5 & - \\
\hline
\end{tabular}

a) All entries are as cited in Refs. [21] and [26]. $\Delta \mathrm{G}^{\dagger}$ calculated at $25^{\circ} \mathrm{C}$. 


\section{3}

\section{Catalytic Mechanism of Chorismate Mutase}

The exact mechanism of the action by chorismate mutase is still not clear, in spite of extensive experimental and theoretical investigations. Several suggestions have been proposed concerning the origin of the catalysis. They include: (a) the stabilization of transition state by the enzyme, presumably through electrostatic interactions from the active site residues; (b) the promotion of substrate conformational transition to generate the reactive CHAIR conformer at the active site (see Scheme 1.2); (c) the increase of populations of near attack conformers (NACs); and (d) strain effects and conformational compression. These proposals will be discussed below.

\subsection{1}

\section{Stabilization of Transition State by Active Site Residues}

The X-ray structures of the CM complexes [4, 6, 7, 12, 34] showed that the enzymes consist of highly charged active site residues and form extensive hydrogen bonding interactions with the inhibitor (Scheme 1.4a-c). Several residues that interact directly with the inhibitor were proved to be crucial for the catalysis from mutagenesis studies (see above). For instance, each enzyme forms two hydrogen bonds with the ether oxygen of TSA. Replacement of the corresponding residue(s) involved the interaction(s) (e.g., Gln88 $\rightarrow$ Ala or Lys39 $\rightarrow$ Ala mutation in EcCM or Arg90 $\rightarrow$ Ala mutation in $\mathrm{BsCM}$ ) and led to a significant reduction of the catalytic efficiency (Table 1.1). Hilvert and his coworkers [36] further showed that in the case of $\mathrm{BsCM}$ the existence of positive charge on Arg90 is very important, as the replacement of Arg90 by a neutral arginine analog (citrulline) causes $>10^{4}$-fold decrease in $k_{\text {cat }}$. These observations have led to the suggestion that one possible origin of the catalysis is electrostatic interactions from the active site residues that stabilize the developing charges in the transition state (TS) and lower the activation barrier.

Several computational studies $[49,58,67,71,75,80]$ of the BsCM catalysis support the suggestion that chorismate mutase works by stabilization of transition state through electrostatic interactions from the active site residues. For instance, Lyne et al. [49], Lee et al. [67] and Ranaghan et al. [76] performed hybrid quantum mechanical/molecular mechanical (QM/MM) studies of BsCM. In a hybrid QM/ MM approach, the flexibility of a quantum mechanical description for a small number of atoms at the active site or in solution (e.g., the atoms in chorismate) is combined with the efficiency of an empirical force field representation for the bulk of the solvated system. So both the chemical events of bond breaking and making (e.g., the change from chorismate to prephenate) as well as the effects of the environment (e.g., the reduction of activation barrier by the enzyme) could be described. Lyne et al. [49], Lee et al. [67] and Ranaghan et al. [76] used a perturbation approach [84] based on fixed BsCM-CHAIR and BsCM-TS structures to study the contribution of each residue to the lowering of the reaction barrier in going 
from the CHAIR conformer to TS. In this perturbation approach, the charges on the atoms of the residue under consideration are turned off. As a result, the reaction barrier would increase (decrease) if that residue stabilizes (destabilizes) the transition state. They showed that, consistent with the experimental observations mentioned above, the deletion of Arg90 led to the increase of the reaction barrier by $3.1-9.6 \mathrm{kcal} / \mathrm{mol}$. Other important residues identified from the perturbation approach include Arg7 and Glu78, and the deletions of these two residues led to increases of the barrier by $1.2-4.0$ and $1.6-3.5 \mathrm{kcal} / \mathrm{mol}$, respectively. The results of these computational studies therefore support the suggestion that the enzyme works by electrostatic stabilization of transition state from the active site residues. Interestingly, it was found from one of the studies [67] that the deletion of Asp118 decreased the reaction barrier by as much as $5 \mathrm{kcal} / \mathrm{mol}$ (corresponding a rate acceleration of more than $10^{3}$-fold), whereas the mutagenesis study showed that the rate is actually lower by a factor of 2 . The perturbation approach has only been used for BsCM. It would be of interest to use this approach to study the EcCM and yeast CM catalysis, where a neutral polar residue (Gln88 in EcCM and Glu246 in yeast CM) interacting with the ether oxygen plays a crucial role in the catalysis.

It should be pointed out that different conclusions may sometime be reached from different calculations. For instance, Worthington et al. [54] showed that the electrostatic stabilization of Arg90 was not stronger for the transition state than for the reactant based on their calculations using the method of effective fragment potentials. The suggestion that the enzyme works by electrostatic stabilization of the transition state is also supported by other computational studies, where the activation barriers in solution and in the enzyme are compared. It was found that there is a significant reduction of the barrier (about $10 \mathrm{kcal} / \mathrm{mol}$ ) in going from solution to the enzyme active site [58, 71, 75, 80]. However, an important factor that needs to be taken into account in this type of studies is that the stable conformations of chorismate in solution and enzyme are different. Such conformational differences may have significant energetic consequences on the activation barriers for the Claisen rearrangement in the different environments. A question that needs to be addressed is whether the reduction of the activation barrier in going from solution to the enzyme active site obtained from computational studies actually comes from the lowering of the TS energy or from some other origin. We will discuss the stabilization of transition state again in connection with other proposals.

\subsection{2}

\section{Substrate Conformational Transition and the Role of Active Site Residues}

Knowles and coworkers [13, 14] demonstrated that the transition state (1 in Scheme 1.2) for the Claisen rearrangement of chorismate has their atoms in the [3,3]-pericyclic region arranged in a chair-like configuration. The pseudodiaxial CHAIR conformer (2 in Scheme 1.2) is the substrate conformation that can reach this chair-like transition state directly. The bond breaking and making process 
may therefore start from CHAIR during the rearrangement. One way for CM to catalyze the reaction is to bind this CHAIR conformer from solution and lower the activation barrier for its chemical transformation at the active site. A requirement for this mechanism is a sufficiently large population of CHAIR in solution. Copley and Knowles [15] performed a NMR study and showed that a pseudodiaxial conformer(s) exists at a reasonable level in water. They assumed that this pseudodiaxial conformer observed in the NMR experiment was CHAIR and suggested that enzyme could bind this reactive conformer directly. Many later discussions of the CM catalysis have been based on this assumption. However, there are different possible pseudodiaxial conformers for chorismate, and these conformers cannot simply be distinguished by the coupling constants measured in the NMR experiment [15]. Moreover, a later study of the transferred nuclear Overhauser effects for chorismate by Hilvert and his coworkers [17] failed to support the original suggestion the stable pseudodiaxial conformer in solution is CHAIR. Consistent with this later experimental study, high level ab initio calculations $[47,48,50$, $68,70,82]$ showed that the reactive CHAIR conformer is considerably less stable than other pseudodiaxial and pseudodiequatorial conformers (see Table 1.3). For instance, the energy difference between CHAIR and the most stable pseudodiequatorial conformer of chorismate can be as much as $18 \mathrm{kcal} / \mathrm{mol}$. It is interesting to note from Table 1.3 that while the results from the SCC-DFTB semiempirical method are rather close to the $a b$ initio results, the AM1 method can overestimate the stability of the CHAIR conformer by as much as $10 \mathrm{kcal} / \mathrm{mol}$ in the gas phase. This suggests that care must be excised in choosing proper computational methods for the study of the Claisen rearrangement of chorismate.

To explore the possible solution conformers, Guo et al. [82] performed QM/MM molecular dynamics simulations using the CHARMM program [86]; the CHAIR

Table 1.3 Energies and structural parameters for some chorismate conformers ${ }^{\mathrm{a}}$.

\begin{tabular}{lllllll}
\hline Conformer & $\begin{array}{l}\Delta E^{\mathrm{b})} \\
\left(\mathbf{k c a l} \cdot \mathrm{mol}^{-1}\right)\end{array}$ & $\begin{array}{l}\boldsymbol{R}_{\mathbf{1}} \\
(\AA)\end{array}$ & $\begin{array}{l}\tau_{1} \\
\text { (degrees) }\end{array}$ & $\begin{array}{l}\tau_{2} \\
\text { (degrees) }\end{array}$ & $\begin{array}{l}\boldsymbol{\tau} \\
\text { (degrees) }\end{array}$ & $\begin{array}{l}\boldsymbol{\delta} \\
\text { (degrees) }\end{array}$ \\
\hline CHAIR & $17.9(13.8)[6.5]$ & 3.8 & -133 & 91 & 73 & 21 \\
DIEQ $_{1}$ & $2.9(3.5)[0.3]$ & 4.4 & -61 & 169 & 79 & -54 \\
DIEQ $_{2}$ & $0.0(0.0)[0.0]$ & 5.3 & -75 & 161 & -106 & -43 \\
DIAX $^{c}$ & & -4.9 & 160 & 71 & -58 & 45 \\
Ex-DIAX & 12.3 & 5.2 & -160 & 71 & -121 & 45 \\
\hline
\end{tabular}

a) Except where otherwise noted, $\Delta \mathrm{E}$ values and the structural parameters are based on B3LYP/6-31G* calculations from Ref. [82]. The energy of $\mathrm{DIEQ}_{2}$ is taken as the zero. $R_{1}=R\left(\mathrm{C}_{1} \ldots \mathrm{C}_{9}\right), \tau_{1}=\tau\left(\mathrm{O}_{7}-\mathrm{C}_{3}-\mathrm{C}_{4}-\mathrm{O}_{12}\right), \tau_{2}=\tau\left(\mathrm{H}-\mathrm{C}_{3}-\mathrm{C}_{4}-\mathrm{H}\right)$, $\tau=\tau\left(\mathrm{C}_{8}-\mathrm{O}_{7}-\mathrm{C}_{3}-\mathrm{C}_{2}\right)$, and $\delta=1 / 2\left(\left|\tau_{1}\right|-\left|\tau_{2}\right|\right)$ (see Scheme 1.5).

b) B3LYP/6-31G* (SCC-DFTB) [AM1].

c) Calculated from the energy minimization at $\mathrm{HF} / 6-31 \mathrm{G} *$ level with eight bridging waters between the two carboxylate groups of chorismate. 
conformer was used as the initial conformation for chorismate. Chorismate was described by QM (SCC-DFTB) [86], and the rest of the system (explicit aqueous solvent) was treated by MM [87]. The quantum mechanical description of the substrate is advantageous because it does not require specific MM parameters to be determined and provides a more realistic treatment of the fluctuations of the covalent bond distances. Although CHAIR is a high energy, local minimum in the gas phase, it is not stable in solution. Instead, it is rapidly (within 10-20 ps) converted to another pseudodiaxial conformer, called DIAX (see Scheme 1.5). Chorismate spends a significant portion of the time in the DIAX conformation during the remainder of the simulations, and the CHAIR conformer was not observed. The values of the dihedral angles describing the ring [i.e., $\tau_{1}\left(\mathrm{O}_{7}-\mathrm{C}_{3}-\mathrm{C}_{4}-\mathrm{O}_{12}\right)$ and $\tau_{2}$ $\left.\left(\mathrm{H}-\mathrm{C}_{3}-\mathrm{C}_{4}-\mathrm{H}\right)\right]$ are similar for DIAX and CHAIR. DIAX is distinguished from CHAIR by $\tau\left(\mathrm{C}_{8}-\mathrm{O}_{7}-\mathrm{C}_{3}-\mathrm{C}_{2}\right)$; i.e., $\tau$ is about $70^{\circ}$ in CHAIR, whereas it is about $-30^{\circ}$ to $-70^{\circ}$ in DIAX. Scheme 1.5 shows that DIAX has a sidechain carboxylate group, rather than the sidechain methylene group, over the $\mathrm{C}_{1}$ atom and is therefore an inactive conformation. However, it may well be the one observed by Copley and Knowles [15] in their solution NMR studies; its structure is consistent with the NMR measurement of Hilvert and coworkers [17]. The results obtained in the solution simulations indicate that the original proposal in which the enzyme preferentially binds the CHAIR conformer is not tenable, because its concentration in solution seems to be too low from the simulations study. Instead, a likely possibility is that DIAX or other conformers, which are relatively stable in solution, are ones bound by the enzyme.

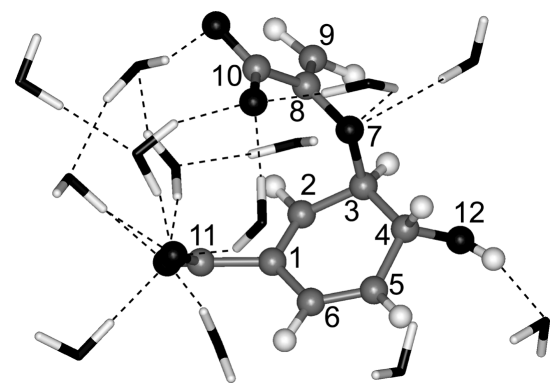

Scheme 1.5 A snapshot of chorismate in solution from the molecular dynamics simulations. The conformation is DIAX with the sidechain carboxylate group over the $\mathrm{C}_{1}$ atom.

The dynamics of CHAIR and DIAX in the active sites of yeast CM [82], BsCM [83] and EcCM (unpublished results) were studied using the QM/MM molecular dynamics simulations, which take into account the motions of the substrate as well as the enzyme. Similar to the solution study, Chorismate was described by QM (SCC-DFTB) [85], and the rest of the system (CM) was treated by MM with the CHARMM force field [88]. It was shown that, in contrast to the motion of CHAIR in solution (see above), no conformational transition occurs in the active sites of the enzymes [82]; the substrate remains in the neighborhood of CHAIR 
during the MD simulations. Moreover, the important interactions with the active site residues observed in the X-ray structures are retained. The stability of CHAIR in the active sites of EcCM and BsCM was confirmed by Hur and Bruice [60-64] from molecular dynamics simulations with a special set of parameters for the substrate (i.e., without the QM description). We will discuss the results of Hur and Bruice later.

For the DIAX conformer, it was shown from the QM/MM MD simulations [8283] that when the inactive conformer is bound to any of the CM active sites, it is rapidly converted to the reactive CHAIR conformer with the important interactions observed in the X-ray structures recovered. Schemes 1.6 and 1.7 show the active site structures before and after the conformational transition for the cases of EcCM and BsCM, respectively; for the structures of the yeast CM complex, (see Ref. [82]). The initial orientation of DIAX in the active sites is such that the sidechain carboxylate group (and the ether oxygen) forms the observed interactions with the active residues. For instance, in the case of BsCM, Arg7 was allowed to form a salt bridge with the sidechain carboxylate group of the substrate in the initial docking configuration, whereas Arg90 formed two hydrogen bonds with the sidechain carboxylate and the ether oxygen, respectively (see Scheme 1.7a). Such initial orientations of the substrate in the active sites are consistent with the results of the experimental studies [22, 23]. Indeed, it was shown that the substrate analogs (e.g., 5 in Scheme 1.3) without the sidechain carboxylate group are not inhibitors or substrates for chorismate mutase [22, 23] (see Section 1.2.2 above), whereas the analogs without the ring carboxylate group can be modest competitive inhibitors. This indicates that the interactions involving the sidechain

a)

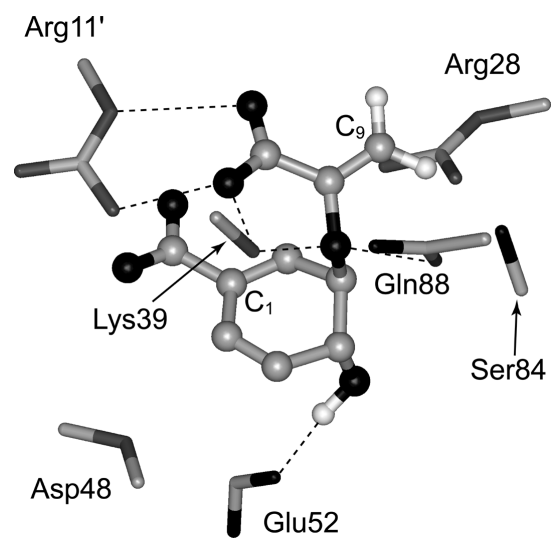

b)

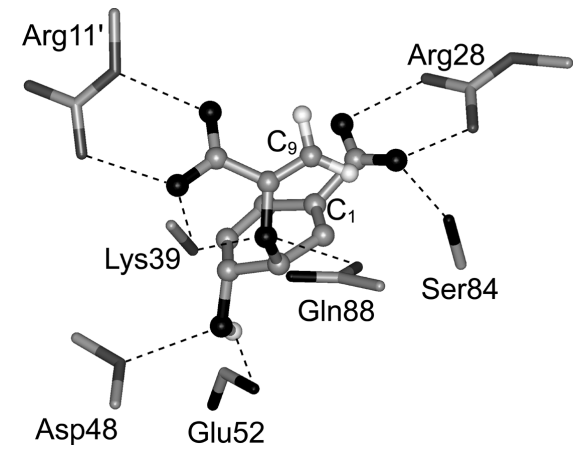

Scheme 1.6 The active site structures before and after the substrate conformational transition for the EcCM-catalyzed reaction. (a) The structure before the transition. The substrate is in the inactive DIAX conformation. (b) The structure after the transition. The substrate is in the CHAIR conformation. 
carboxylate group play a very important role for the binding process [2]. As is evident from Schemes 1.6 and 1.7, the ring of chorismate undergoes a significant clockwise rotation in the active sites of $\mathrm{EcCM}$ and $\mathrm{BsCM}$ during the MD simulations, and such rotation generates the reactive CHAIR conformation from the solution DIAX conformation.

For EcCM, Arg28, Ser84 and the backbone amide group of Asp48 seem to provide the main driving forces for the rotation of the ring of the substrate (Scheme 1.6). Glu52 already forms the hydrogen bond with the $\mathrm{C}_{4}$-hydroxyl group before the rotation and is unlikely to play a role for the conformational transition. By contrast, Glu78 in BsCM does not form the hydrogen bond with the $\mathrm{C}_{4}$-hydroxyl group of DIAX, and this hydrogen bond is formed only when the substrate changes to CHAIR (Scheme 1.7b). Thus, unlike the case involving Glu52 in EcCM, the negatively charged field from Glu78 and the potential hydrogen bonding interaction with the $\mathrm{C}_{4}$-hydroxyl group may make a significant contribution to the rotation of the ring during the conformational transition. It is interesting to note that the results of the MD simulations seem to be consistent with the mutagenesis studies (Table 1.1), where it was shown that the existence of the carboxylate group in the vicinity of the $\mathrm{C}_{4}$-hydroxyl is more important for the BsCM-catalyzed reaction than for the EcCM-catalyzed reaction. Another residue that might also provide the driving force for the conformational transition is Arg63', although the mutagenesis study for the Arg63 mutants has not been available. Conformational transitions from another inactive pseudodiequatorial conformer $\left(\mathrm{DIEQ}_{1}\right)$ to CHAIR were also observed in the active sites $[60,82]$. These results from the QM/ MM MD simulations suggest that one contribution of the enzyme is to bind the
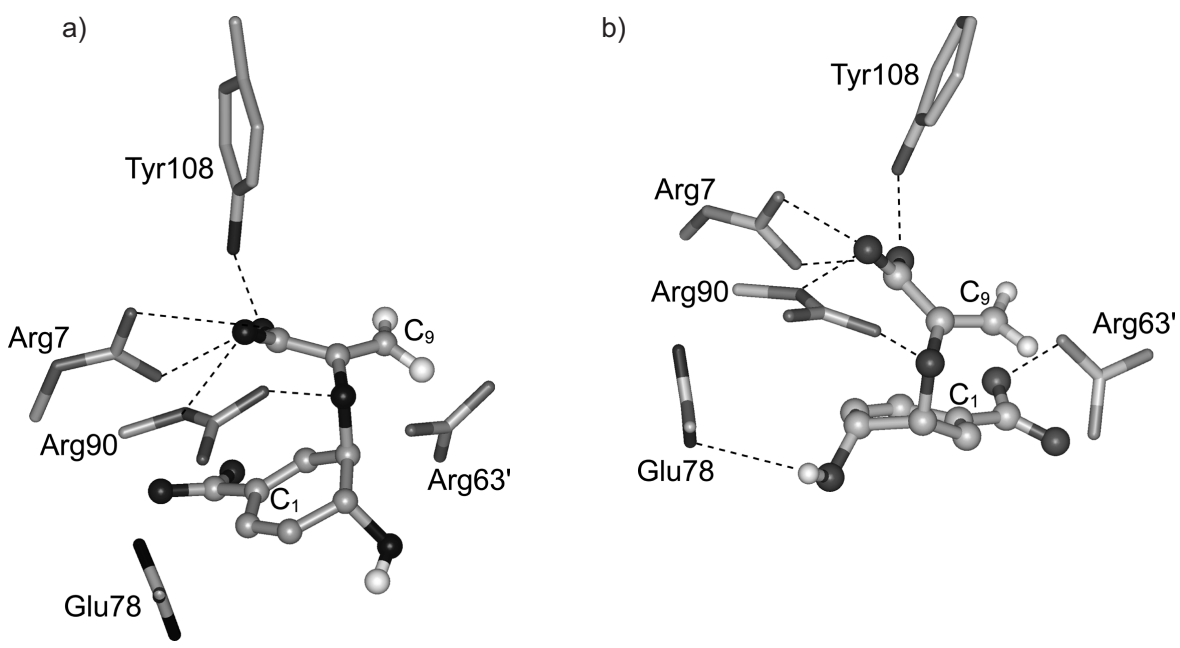

Scheme 1.7 The active site structures before and after the substrate conformational transition for the BsCM-catalyzed reaction. (a) The structure before the transition. The substrate is in the DIAX conformation.

(b) The structure after the transition. The substrate is in the CHAIR conformation. 
more prevalent nonreactive conformers and transform them into the active form in a step before the chemical reaction. This suggestion seems to be supported by the studies of substrate structural requirements for catalysis (Section 1.2.2). Indeed, it was shown that $\mathrm{EcCM}$ and $\mathrm{BsCM}$ are unable to catalyze the rearrangement of $\mathbf{6}$ (which lacks the ring carboxyl group) [22, 23], even though $\mathbf{6}$ is an inhibitor for the both enzymes. The deletion of the ring carboxylate group would remove some of the important driving forces required for the conformational transition (e.g., the interaction between Arg28 and the ring carboxylate) and destroy the ability of the enzyme to generate the reactive CHAIR conformation in the active site, leading a reduction of the catalytic efficiency.

To examine the free energy involved in the stabilization of the CHAIR form in the active site and the role of the active site residues, the QM/MM molecular dynamics simulations and umbrella sampling simulations were performed for the wild-type $\mathrm{BsCM}$ as well as mutant enzymes [83]. This study showed that Arg90 is essential for stabilizing the reactive substrate conformation (CHAIR) in the active site, as both the Arg90Ala and Arg90Gly mutations destroy the ability of the wild-type enzyme to stabilize CHAIR and stabilize the inactive DIAX conformation instead. CHAIR was found to be about $10 \mathrm{kcal} / \mathrm{mol}$ more stable than DIAX in the wild-type BsCM, and it becomes $5 \mathrm{kcal} / \mathrm{mol}$ less stable in Arg90Ala. The results of the MD and free energy simulations therefore suggested an additional role for Arg90. Its existence appears to be essential for generating and stabilizing the CHAIR conformation of the substrate in the active site. The extra energy cost $(\sim 5 \mathrm{kcal} / \mathrm{mol})$ necessary for the formation of CHAIR in Arg90Ala and Arg90Gly obtained from the simulations would contribute to the loss of activity of the mutants [31]. Thus, the stabilization of the chair-like conformations of chorismate and the transition state [25] seems to require a proper balance of different interactions at the active site. The removal of the single key residue, such as Arg90, can destroy such delicate balance of the interactions, leading to a dramatic reduction in the catalytic efficiency.

The mutagenesis study [31] showed that the double mutant Cys88Lys/Arg90Ser and Cys88Ser/Arg90Lys can restore a factor of more than $10^{3}$ in $k_{\text {cat }}$ (see above). This restoration of the catalytic efficiency may be explained by a similar argument. That is, the relatively high activities for Cys88Lys/Arg90Ser and Cys88Ser/ Arg90Lys could be related to the more balanced interactions generated by the double mutations that are able to stabilize a substrate conformation close to CHAIR. Indeed, the CHAIR conformer was found to be more stable in the double mutant Cys88Lys/Arg90Ser than Arg90Ala from the free energy simulations [83]. In the Cys88Lys/Arg90Ser mutant, the substrate is somewhat distorted, and additional energy (circa $2 \mathrm{kcal} / \mathrm{mol}$ ) is necessary to generate the optimal reactive CHAIR conformation. This factor, as well as the lack of a stable hydrogen-bonding interaction with the ether oxygen atom, would make the double mutants less efficient than the wild-type enzyme ( $k_{\text {cat }}$ values are about $10^{2}$-fold less than that of wild-type). 


\section{3 .3}

\section{Contribution of the Near Attack Conformers (NACs)}

The concept of the near attack conformers $[89,90]$ has also been applied to the mechanism of the CM-catalyzed reaction by Hur and Bruice [60-64]. The near attack conformers (NACs) are special substrate conformations in which the bondforming atoms are at the van der Waals distance and at an angle near the one in the transition state [60-64]. One convenience of the NAC approach in understanding enzymes is that quantum mechanical calculations may not be necessary for the description of chemical process of bond breaking and making. Therefore, one only needs to focus on the geometrical arrangements of the atoms in the substrate that are involved in the bond formation. However, it has been pointed out $[80,91]$ that one intrinsic problem with this concept is that there does not seem to be a unique way of defining NAC. This is in contrast with the concept of the transition state. A transition state is a well-defined stationary point on the surface and satisfies a variational criterion [91]. According to the results based on the NAC approach, chorismate mutase speeds up the Claisen rearrangement by binding its substrate primarily in its NAC form, rather than by lowering the transition-state barrier [61-62]. However, as Garcia-Viloca et al. [91] pointed out, the essential NAC effect is likely to be that "the activation free energy barrier is reduced by the substrate conformational change induced by the enzyme" (see above).

Different definitions for NCA have been used in the study of the Claisen rearrangement of chorismate [60-64, 77-80]. Some of the inconsistencies in the results as well as in the interpretations might be related to the use of the different definitions (see below). In the earlier studies [60, 62-64], Hur and Bruice used two geometrical parameters to define NAC, and the parameters include the distance between $\mathrm{C}_{1}$ and $\mathrm{C}_{9}\left(R_{1}\right)$ and the attacking angle $\theta_{1}$ (see Scheme 1.8a). A conformation of chorismate was considered to be NAC if $R_{1} \leq 3.7 \AA$ and $\theta_{1} \leq 40^{\circ}$ [60]. In some other investigations [77-78], only the condition on $R_{1}$ (i.e., $R_{1} \leq 3.7 \AA$ ) was used in the definition of NAC. In a more recent study, Hur and Bruice [61] included an additional parameter, $\theta_{2}$, in their definition of NAC (see Scheme $1.8 \mathrm{~b}$ ) and suggested that the three conditions for being NAC are: (1) $R_{1} \leq 3.7 \AA$, (2) $\theta_{1} \leq 28.2^{\circ}$ and (3) $\theta_{2} \leq 38.2^{\circ}$. It should be mentioned that the NACs defined by the single parameter $\left(R_{1}\right)$, the two parameters $\left(R_{1}\right.$ and $\left.\theta_{1}\right)$ or even three parameters $\left(R_{1}, \theta_{1}\right.$ and $\left.\theta_{2}\right)$ may not correspond to the reactive CHAIR conformation (see Scheme 1.2). As we mentioned earlier, the CHAIR conformer of the substrate can change to the chair-like transition state directly with the $\mathrm{C}_{1}$ and $\mathrm{C}_{9}$ carbon atoms well positioned to form the carbon-carbon bond. The conformers that satisfy the NAC conditions may not posses these properties. To demonstrate this point, Scheme 1.9a and 1.9b show two typical conformations of chorismate in solution that were observed during the QM/MM MD simulations using AM1 method [93] (Guo et al., unpublished results; see also Ref. [61]). The conformation in Scheme 1.9a satisfies the condition on $R_{1}$ (i.e., $R_{1}=3.55 \AA$. Also, $\theta_{1}$ is only $2.6^{\circ}$ greater than $40^{\circ}$ ). Nevertheless, the conformer is inactive because the $\pi$ orbital of $\mathrm{C}_{9}$ is not positioned to form the carbon-carbon bond with $\mathrm{C}_{1}$. The conformation in Scheme 1.9b 
a)

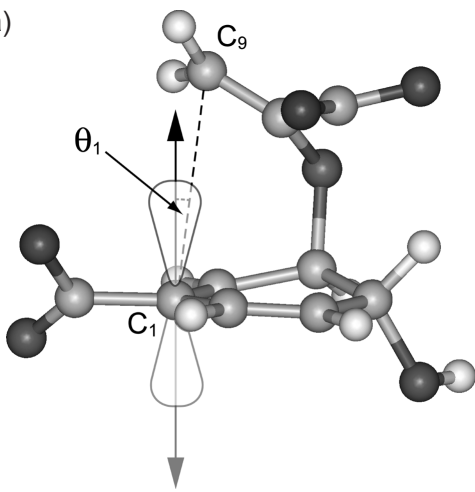

Scheme 1.8

a)

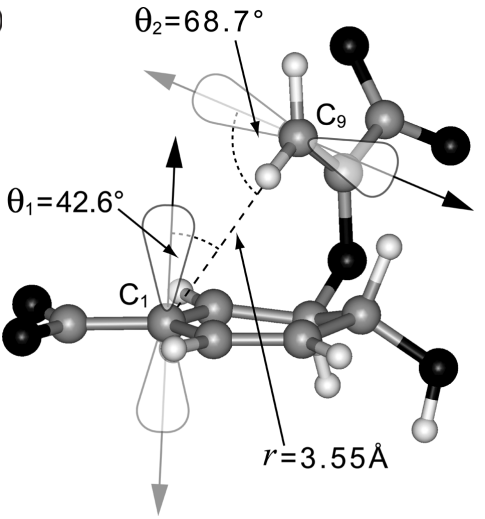

b)

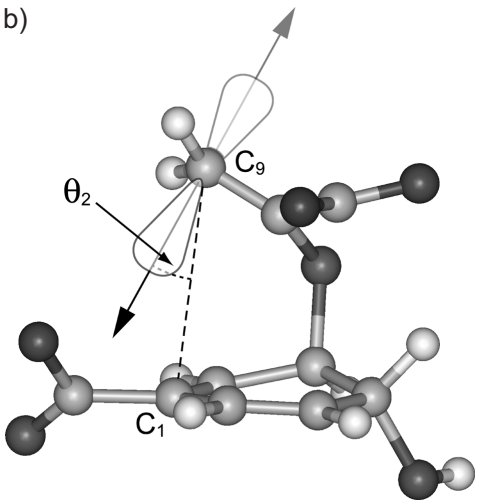

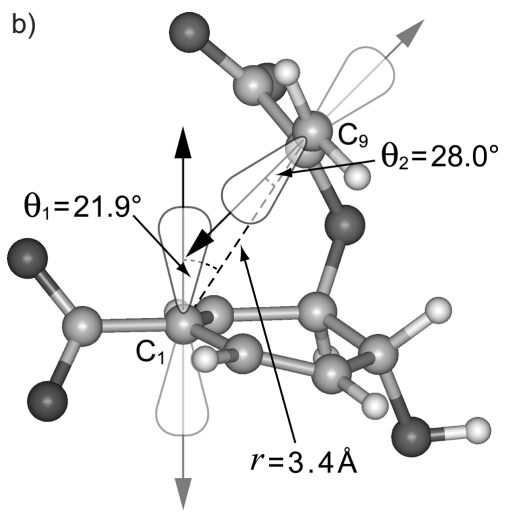

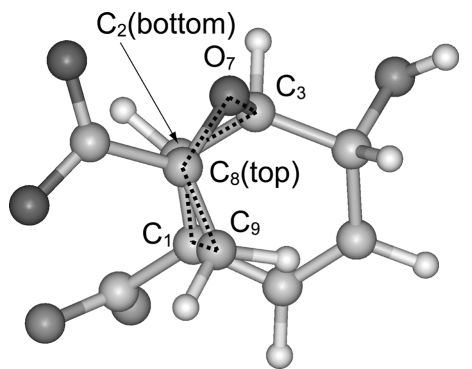

Scheme 1.9 Two typical conformations observed in the simulations of chorismate in solution using the AM1 method for chorismate.

(a) An inactive conformation that satisfies $R_{1} \leq 3.7 \AA$. (b) A "boat-like" NAC that satisfies all the three conditions for NAC proposed in Ref. [61]. 
satisfies all the three conditions proposed by Hur and Bruice (i.e., $R_{1} \leq 3.7 \AA$, $\theta_{1} \leq 28.2^{\circ}$ and $\left.\theta_{2} \leq 38.2^{\circ}\right)$. However, this conformer will not lead to the chair-like transition state. As we mentioned earlier, it has been demonstrated by Knowles and coworkers $[13,14]$ that the Claisen rearrangement of chorismate to prephenate proceeds through the chair-like transition state in solution and enzyme active site. In fact, the NAC in Scheme 1.9b will lead to a "boat-like" transition state in which the atoms in the [3,3]-pericyclic region are arranged in a boat-like configuration. Because the reaction does not proceed through the boat-like transition state either in solution or in the enzyme active site, the formation of the boat-like NACs may not be of great interest. The key point in the NAC concept is that the geometrical parameters for the atoms associated with the bond formation are close to those in the transition state. For the Claisen rearrangement, these geometrical parameters are similar in the chair-like and boat-like transition states. Therefore, the use of these parameters alone is not sufficient to distinguish the boat-like NAC from the chair-like NAC (which is essentially the reactive CHAIR conformation).

Hur and Bruice [61-63] considered the formation of the transition state as two different stages, namely, the formation of NAC with a free energy of formation of $\Delta G_{\mathrm{N}}^{\circ}$ and the formation of the transition state from NAC with a free energy of $\Delta G_{\mathrm{Ts}}^{\ddagger}$ :

$$
\Delta G^{\dagger}=\Delta G_{\mathrm{N}}^{\circ}+\Delta G_{\text {†s }}^{\dagger}
$$

Here $\Delta G^{t}$ is the activation energy for the Claisen rearrangement. Thus, important insights concerning the origin of the catalysis may be obtained by examining

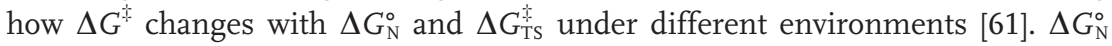
can be estimated from the probability of occurring in molecular dynamics simulations [63] or from free energy simulations, whereas $\Delta G_{\text {TS }}^{\ddagger}$ may be obtained from QM/MM calculations. Hur and Bruice [61] performed molecular dynamics simulations using the CHARMM program with a special (unpublished) set of parameters for chorismate. They estimated $\Delta G_{\mathrm{N}}^{\circ}$ in different environments (solution, 1F7 catalytic antibody, the R90Cit mutant of BsCM, the E52A mutant of EcCM, wild-type BsCM, and wild-type EcCM) and plotted the experimental $\Delta G^{\dagger}$ values as a function of $\Delta G_{N}^{\circ}$. It was found that $\Delta G^{t}$ changes with $\Delta G_{N}^{\circ}$ linearly with a slope of 1.1. Moreover, the $\Delta G_{\mathrm{TS}}$ values obtained by subtracting $\Delta G_{\mathrm{N}}^{\circ}$ from the experimental $\Delta G^{\dagger}$ values are essentially a constant (see Table 1.4) in the different environments, as one would expect based on the linear relationship between $\Delta G^{\dagger}$ and $\Delta G_{\mathrm{N}}^{\circ}$. These data support the proposal that the relative rate of the Claisen rearrangement overwhelmingly depends on the efficiency of formation of NAC [61]. Although the results of Hur and Bruice are of considerable interest, there are some significant inconsistencies with other computational studies. Several investigations have suggested that the rate acceleration by chorismate mutase is mainly due to the electrostatic stabilization of the transition state at the enzyme active site $[49,58,67,71,75,80]$ and that the efficiency of formation of NAC should not be considered as the major origin for the catalysis [77-78, 80]. For instance, Štrajbl et al. [80] performed empirical valence bond (EVB) and binding free energy calcu- 
Table 1.4 Relationship between the free energies in different environments ${ }^{\mathrm{a}}$.

\begin{tabular}{|c|c|c|c|c|c|}
\hline Environment & $\Delta G$ & - & $\Delta G_{N}^{\circ}$ & $=$ & $\Delta G_{\mathrm{Ts}}$ \\
\hline Solution & 24.2 & & 8.1 & & 16.1 \\
\hline Wild type EcCM & 15.2 & & 0.1 & & 15.1 \\
\hline Wild type BsCM & 15.4 & & 0.3 & & 15.1 \\
\hline E52A (EcCM) & 18.2 & & 1.3 & & 16.9 \\
\hline R90Cit (BsCM) & 21.2 & & 4.1 & & 17.1 \\
\hline $1 \mathrm{~F} 7$ & 21.3 & & 5.5 & & 15.8 \\
\hline
\end{tabular}

a) From Ref. [61] and in $\mathrm{kcal} \cdot \mathrm{mol}^{-1}$.

lations. They showed that the major catalysis effect of chorismate mutase is electrostatic transition state stabilization (TSS). Although Štrajbl et al. also obtained the NAC effect of about $5 \mathrm{kcal} / \mathrm{mol}$, they suggested that this effect was not the reason for the catalysis but the result of the TSS.

There are different possible reasons for the inconsistencies. For instance, different definitions for NAC have been used in different investigations that could result in discrepancies in the free energy of formation for NAC. For instance, the NACs defined only by $R_{1} \leq 3.7 \AA$ would lead to a smaller free energy of conformation for NAC than that defined by the three conditions. This is because more conformations (e.g., the conformation in Scheme 1.9a) would be counted as NACs. In the analysis of the origin of enzyme catalysis based on the NAC concept, the effect of the enzyme on the activation barrier is divided into the two different contributions (see above). An underestimate (overestimate) of the free energy for the NAC formation would result in an overestimate (underestimate) of $\Delta \mathrm{G}_{\mathrm{TS}}^{\mathrm{t}}$, leading to a different conclusion concerning the importance of the NAC formation. Thus, a consistent definition of NAC should be very important for the comparison of different simulations results. Another possible reason for the inconsistencies would be the use of different computational approaches and force field parameters. For instance, the AM1 method has been widely used in the study of the Claisen rearrangement of chorimate in solution and enzyme. However, the gas phase calculations showed that the AM1 method overestimates the stability of the CHAIR conformer significantly (see Table 1.3). Such deficiencies in the AM1 method would affect the accuracy of the simulation results as well as the conclusion concerning the origin of the catalysis.

\subsection{4}

\section{Strain Effects and Conformational Compression}

Strains and conformational compression of the substrate at the enzyme active site were also proposed to explain the rate acceleration by chorismate mutase $[66,70$, 
78]. For instance, Jorgensen and his coworkers [78] suggested, based on the results of their calculations and analysis, that the stabilization of the transition state in chorismate mutase plays only a secondary role and that the rate enhancement is primarily due to conformational compress of the substrate. However, the analysis of Jorgensen and coworkers has been called into question [80], and it was shown that the results might be interpreted differently [80].

\section{4}

\section{Conclusion}

The discussions given in this chapter have shown that although chorismate mutase has been a subject of extensive experimental and theoretical investigations, there are still considerable uncertainties concerning how the Claisen rearrangement from chorismate to prephenate is actually catalyzed by the enzyme. The computational investigations have led different possibilities, but experimental studies with modern techniques are necessary to identify the most likely mechanism of the CM catalysis.

\section{Acknowledgment}

The work described herein was supported by the Center of Excellence for Structural Biology, University of Tennessee. We thank Prof. Martin Karplus for insightful discussions.

\section{List of Abbreviations}

$\begin{array}{ll}\text { BsCM } & \begin{array}{l}\text { Bacillus subtillus chorismate mutase } \\ \text { chair-like configuration } \\ \text { CHAIR }\end{array} \\ \text { CM } & \text { pserismate-pyruvate mutase, EC 5.4.99.5 } \\ \text { DIAX } & \text { inactive pseudodiequatorial conformer } \\ \text { DIEQ } & \text { Escherichia coli chorismate mutase from P-protein } \\ \text { EcCM } & \text { empirical valence bond } \\ \text { EVB } & \text { molecular dynamics } \\ \text { MD } & \text { molecular mechanics } \\ \text { MM } & \text { near attack conformers } \\ \text { NAC } & \text { self-consistent charge density functional tight binding } \\ \text { SCC-DFTB } & \text { transition state } \\ \text { TS } & \text { transition state analog }\end{array}$




\section{References}

1 Haslam, E. Shikimic Acid Metabolism and Metabolites, John Wiley \& Sons, New York, 1993.

2 Lee, A. Y.; Stewart, J. D.; Clardy, J.; Ganem, B. Chem. Biol. 1995, 2, 195-203.

3 Ganem, B. Angew. Chem. Int. Ed. 1996, 35, 936-945.

4 Lee, A. Y.; Karplus, P. A.; Ganem, B.; Clardy, J. J. Am. Chem. Soc. 1995, 117, 3627-3628.

5 Stewart, J.; Wilson, D. B.; Ganem, B. J. Am. Chem. Soc. 1990, 112, 4582-4584.

6 Chook, Y. M.; Ke, H. M.; Lipscomb, W. N. Proc. Nat. Acad. Sci. USA. 1993, 90, 8600-8603.

7 Chook, Y. M.; Gray, J. V.; Ke, H. M.; Lipscomb, W. N. J. Mol. Biol. 1994, 240, 476-500.

8 Ladner, J. E.; Reddy, P.; Davis, A.; Tordova, M.; Howard, A. J.; Gilliland, G. L. Acta Crystallogr. Sect. D Biol. Crystallogr. 2000, 56, 673-683.

9 Hilvert, D.; Carpenter, S. H.; Nared, K. D.; Auditor, M. T. M. Proc. Nat. Acad. Sci. USA 1988, 85, 4953-4955.

10 Xue, Y. F.; Lipscomb, W. N.; Graf, R.; Schnappauf, G.; Braus, G. Proc. Nat. Acad. Sci. USA 1994, 91, 10814-10818.

11 Xue, Y. F.; Lipscomb, W. N. J. Mol. Biol. 1994, 241, 273-274.

12 Strater, N.; Hakansson, K.; Schnappauf, G.; Braus, G.; Lipscomb, W. N. Proc. Nat. Acad. Sci. USA 1996, 93, 3330-3334.

13 Sogo, S. G.; Widlanski, T. S.; Hoare, J. H.; Grimshaw, C. E.; Berchtold, G. A.; Knowles, J. R. J. Am. Chem. Soc. 1984 , 106, 2701-2703.

14 Copley, S. D.; Knowles, J. R. J. Am. Chem. Soc. 1985, 107, 5306-5308.

15 Copley, S. D.; Knowles, J. R. J. Am. Chem. Soc. 1987, 109, 5008-5013.

16 Andrews, P. R.; Haddon, R. C. Aust. J. Chem. 1979, 32, 1921-1929.

17 Campbell, A. P.; Tarasow, T. M.; Massefski, W.; Wright, P. E.; Hilvert, D. Proc. Nat. Acad. Sci. USA 1993, 90, 8663-8667.

18 Clarke, T.; Stewart, J. D.; Ganem, B. Tetrahedron 1990, 46, 731-748.
19 Bartlett, P. A.; Nakagawa, Y.; Johnson, C. R.; Reich, S. H.; Luis, A. J. Org. Chem. 1988, 53, 3195-3210.

20 Andrews, P. R.; Smith, G. D.; Young, I. G. Biochemistry 1973, 12, 3492-3498.

21 Galopin, C. C.; Zhang, S.; Wilson, D. B.; Ganem, B. Tetrahedron Lett. 1996, 37, 8675-8678.

22 Pawlak, J. L.; Padykula, R. E.; Kronis, J. D.; Aleksejczyk, R. A.; Berchtold, G. A. J. Am. Chem. Soc. 1989, 111, 3374-3381.

23 Galopin, C. C.; Ganem, B. Bioorg. Med. Chem. Lett. 1997, 7, 2885-2886.

24 Crespo, A.; Scherlis, D. A.; Marti, M. A.; Ordejon, P.; Roitberg, A. E.; Estrin, D. A. J. Phys. Chem. B 2003, 107, 13728-13736.

25 Cload, S. T.; Liu, D. R.; Pastor, R. M.; Schultz, P. G. J. Am. Chem. Soc. 1996, 118, 1787-1788.

26 Kast, P.; AsifUllah, M.; Hilvert, D. Tetrahedron Lett. 1996, 37, 2691-2694.

27 Liu, D. R.; Cload, S. T.; Pastor, R. M.; Schultz, P. G. J. Am. Chem. Soc. 1996, 118, 1789-1790.

28 Schnappauf, G.; Strater, N.; Lipscomb, W. N.; Braus, G. H. Proc. Nat. Acad. Sci. USA 1997, 94, 8491-8496.

29 Kast, P.; Hartgerink, J. D.; AsifUllah, M.; Hilvert, D. J. Am. Chem. Soc. 1996, 118, 3069-3070.

30 Kast, P.; AsifUllah, M.; Jiang, N.; Hilvert, D. Proc. Nat. Acad. Sci. USA 1996, 93, 5043-5048.

31 Kast, P.; Grisostomi, C.; Chen, I. A.; Li, S. L.; Krengel, U.; Xue, Y. F.; Hilvert, D. J. Biol. Chem. 2000, 275, 36832-36838.

32 Ife, R.; Ball, L. F.; Lowe, P.; Haslam, E. J. Chem. Soc. Perkin Trans. I 1976, 1776-1783.

33 Haynes, M. R.; Stura, E. A.; Hilvert, D.; Wilson, I. A. Science 1994, 263, 646-652.

34 Strater, N.; Hakansson, K.; Schnappauf, G.; Braus, G.; Lipscomb, W. N. Structure 1997, 5, 1437-1452.

35 Zhang, S.; Kongsaeree, P.; Clardy, J.; Wilson, D. B.; Ganem, B. Bioorg. Med. Chem. 1996, 4, 1015-1020.

36 Kienhofer, A.; Kast, P.; Hilvert, D. J. Am. Chem. Soc. 2003, 125, 3206-3207. 
37 Zhang, S.; Pohnert, G.; Kongsaeree, P.; Wilson, D. B.; Clardy, J.; Ganem, B. J. Biol. Chem. 1998, 273, 6248-6253.

38 Gustin, D. J.; Mattei, P.; Kast, P.; Wiest, O.; Lee, L.; Cleland, W. W.; Hilvert, D. J. Am. Chem. Soc. 1999, 121, 1756-1757.

39 Lee, A.L.; Zhang S.; Kongsaeree; Clardy, J.; Ganem, B.; Erickson, J. W.; Xie, D. Biochemistry 1998, 37, 9052-9057.

40 Addadi, L.; Jaffe, E. K.; Knowles, J. R. Biochemistry 1983, 22, 4494-4501.

41 Andrews, P. R.; Cain, E. N.; Rizzardo, E.; Smith, G. D. Biochemistry 1977, 16, 4848-4852.

42 Gorisch, H. Biochemistry 1978, 17, 3700-3705.

43 Gamper, M.; Hilvert, D; Kast, P. Biochemistry, 2000, 39, 14087-14094.

44 Ma, J. P.; Zheng, X. F.; Schnappauf, G.; Braus, G.; Karplus, M.; Lipscomb, W. N. Proc. Nat. Acad. Sci. USA 1998, 95, 14640-14645.

45 Sturtevant, J. M. Proc. Nat. Acad. Sci. USA 1977, 74, 2236-2240.

46 Naghibi, H.; Tamura, A.; Sturtevant, J. M. Proc. Nat. Acad. Sci. U.S.A. 1995, 92, 5597-5599.

47 Wiest, O.; Houk, K. N. J. Am. Chem. Soc. 1995, 117, 11628-11639.

48 Wiest, O.; Houk, K. N. J. Org. Chem. 1994, 59, 7582-7584.

49 Lyne, P. D.; Mulholland, A. J.; Richards, W. G. J. Am. Chem. Soc. 1995, 117, 11345-11350.

50 Davidson, M. M.; Gould, I. R.; Hillier, I. H. J. Chem. Soc., Chem. Commun. 1995, 63-64.

51 Davidson, M. M.; Gould, I. R.; Hillier, I. H. J. Chem. Soc., Perkin Trans. 2 1996, 525-532.

52 Davidson, M. M.; Guest, J. M.; Craw, J. S.; Hillier, I. H.; Vincent, M. A. J. Chem. Soc., Perkin Trans. 2 1997, 1395-1400.

53 Worthington, S. E.; Krauss, M. J. Phys. Chem. B 2001, 105, 7096-7098.

54 Worthington, S. E.; Roitberg, A. E.; Krauss, M. J. Phys. Chem. B 2001, 105, 7087-7095.

55 Worthington, S. E.; Roitberg, A. E.; Krauss, M. Int. J. Quantum Chem. 2003, 94, 287-292.

56 Hall, R. J.; Hindle, S. A.; Burton, N. A.; Hillier, I. H. J. Comput. Chem. 2000, 21, 1433-1441.
57 Carlson, H. A.; Jorgensen, W. L. J. Am. Chem. Soc. 1996, 118, 8475-8484.

58 Crespo, A.; Scherlis, D. A.; Marti, M. A.; Ordejon, P.; Roitberg, A. E.; Estrin, D. A. J. Phys. Chem. B 2003, 107, 13728-13736.

59 Guimaraes, C. R. W.; Repasky, M. P.; Chandrasekhar, J.; Tirado-Rives, J.; Jorgensen, W. L. J. Am. Chem. Soc. 2003, 125, 6892-6899.

60 Hur, S.; Bruice, T. C. Proc. Nat. Acad. Sci. USA 2002, 99, 1176-1181.

61 Hur, S.; Bruice, T. C. Proc. Nat. Acad. Sci. USA 2003, 100, 12015-12020.

62 Hur, S.; Bruice, T. C. J. Am. Chem. Soc. 2003, 125, 10540-10542.

63 Hur, S.; Bruice, T. C. J. Am. Chem. Soc. 2003, 125, 5964-5972.

64 Hur, S.; Bruice, T. C. J. Am. Chem. Soc. 2003, 125, 1472-1473.

65 Kast, P.; Tewari, Y. B.; Wiest, O.; Hilvert, D.; Houk, K. N.; Goldberg, R. N. J. Phys. Chem. B 1997, 101, 10976-10982.

66 Khanjin, N. A.; Snyder, J. P.; Menger, F. M. J. Am. Chem. Soc. 1999, 121, 11831-11846.

67 Lee, Y. S.; Worthington, S. E.; Krauss, M.; Brooks, B. R. J. Phys. Chem. B 2002, 106, 12059-12065.

68 Madurga, S.; Vilaseca, E. Phys. Chem. Chem. Phys. 2001, 3, 3548-3554.

69 Madurga, S.; Vilaseca, E. J. Phys. Chem. A 2002, 106, 11822-11830.

70 Marti, S.; Andres, J.; Moliner, V.; Silla, E.; Tunon, I.; Bertran, J. J. Phys. Chem. B 2000, 104, 11308-11315.

71 Marti, S.; Andres, J.; Moliner, V.; Silla, E.; Tunon, I.; Bertran, J. Theor. Chem. Acc. 2001, 105, 207-212.

72 Marti, S.; Andres, J.; Moliner, V.; Silla, E.; Tunon, I.; Bertran, J. Chem. Eur. J. 2003, 9, 984-991.

73 Marti, S.; Andres, J.; Moliner, V.; Silla, E.; Tunon, I.; Bertran, J. Theochem. J. Mol. Struct. 2003, 632, 197-206.

74 Marti, S.; Andres, J.; Moliner, V.; Silla, E.; Tunon, I.; Bertran, J. J. Am. Chem. Soc. 2004, 126, 311-319.

75 Marti, S.; Andres, J.; Moliner, V.; Silla, E.; Tunon, I.; Bertran, J.; Field, M. J. J. Am. Chem. Soc. 2001, 123, 1709-1712.

76 Ranaghan, K. E.; Ridder, L.; Szefczyk, B.; Sokalski, W. A.; Hermann, J. C.; Mulholland, A. J. Mol. Phys. 2003, 101, 2695-2714. 
77 Repasky, M. P.; Guimaraes, C. R. W.; Chandrasekhar, J.; Tirado-Rives, J.; Jorgensen, W. L. J. Am. Chem. Soc. 2003, 125, 6663-6672.

78 Guimaraes, C. R. W.; Repasky, M. P.; Chandrasekhar, J.; Tirado-Rives, J.; Jorgensen, W. L. J. Am. Chem. Soc. 2003, 125, 6892-6899.

79 Roca, M.; Marti, S.; Andres, J.; Moliner, V.; Tunon, M.; Bertran, J.; Williams, A. H. J. Am. Chem. Soc. 2003, 125, 7726-7737.

80 Strajbl, M.; Shurki, A.; Kato, M.; Warshel, A. J. Am. Chem. Soc. 2003, 125, 10228-10237.

81 Woodcock, H. L.; Hodoscek, M.; Sherwood, P.; Lee, Y. S.; Schaefer, H. F.; Brooks, B. R. Theor. Chem. Acc. 2003, 109, 140-148.

82 Guo, H.; Cui, Q.; Lipscomb, W. N.; Karplus, M. Proc. Nat. Acad. Sci. USA 2001, 98, 9032-9037.

83 Guo, H.; Cui, Q.; Lipscomb, W. N.; Karplus, M. Angew. Chem. Int. Ed. 2003, 42, 1508-1511.

84 (a) Schowen, R. L. Proc. Nat. Acad. Sci. USA 2003, 100, 11931-11932. (b) Chem. Eng. News 2004, 82, 35-39.

85 Brooks, B.; Bruccoleri, R. E.; Olafson, B. D.; States, D. J.; Swaminathan, S.; Karplus, M. J. Comput. Chem. 1983, 4, 187.
86 (a) Elstner, M.; Porezag, D.; Jungnickel, G.; Elsner, J.; Haugk, M.; Frauenheim, T.; Suhai, S.; Seigert, G. Phys. Rev. B 2001, 58, 7260-7268.

(b) Cui, Q.; Elstner, M.; Kaxiras, E.; Frauenheim, T.; Karplus, M. J. Phys. Chem. B 2001, 105, 569-585.

87 (a) Jorgensen, W. L. J. Am. Chem. Soc. 1981, 103, 335. (b) Neria, E.; Fischer, S.; Karplus, M. J. Chem. Phys. 1996, 105, 1902-1921.

88 MacKerell, A. D. Jr.; Bashford, D.; Bellott, M.; Dunbrack, R. L.; Evanseck, J. D.; Field, M. J.; Fischer, S.; Gao, J.; Guo, H.; Ha, S.; Joseph-McCarthy, D.; Kuchnir, L.; Kuczera, K.; Lau, F. T. K.; Mattos, C.; Michnick, S.; Ngo, T.; Nguyen, D. T.; Prodhom, B.; Reiher, W. E.; Roux, B.; Schlenkrich, M.; Smith, J. C.; Stote, R.; Straub, J.; Watanabe, M.; WiorkiewiczKuczera, J.; Yin, D.; Karplus, M. J. Phys. Chem. B 1998, 102, 3586-3616.

89 Bruice, T. C. Acc. Chem. Res. 2002, 35, 139-148.

90 Bruice, T. C.; Benkovic, S. J. Biochemistry 2000, 39, 6267.

91 Garcia-Viloca, M.; Gao, J.; Karplus, M.; Truhlar, D. G. Science 2004, 303, 186-195. 92 Dewar, M. J. S.; Zoebisch, E. G.; Healy, E. F.; Steward, J. J. P. J. Am. Chem. Soc. 1985, 107, 3902-3909. 
